\section{[gw22-e0809] INDIVIDUALISED STRATEGY OF WARFARIN TREATMENT FOR DEEP VEIN THROMBOEMBOLISM - CASE REPORTS}

Nan Li', Li-zheng Fang², Hong-lei Dai ${ }^{3}$, Jonathan Lio ${ }^{4}$, Li-ying Chen ${ }^{5}$, Ke-jing Ying ${ }^{6}{ }^{1}$ Department of Medicine, University of Hong Kong; ${ }^{2,3,5}$ Department of Family Physician, Sir Run Run Shaw Hospital, Zhejiang University; ${ }^{4}$ Department of Medicine, Johns Hopkins Bayview Hospital, ' ${ }^{6}$ Department of Medicine, Sir Run Run Shaw Hospital, Zhejiang University

\subsection{6/heartjnl-2011-300867.557}

Warfarin sodium (Coumadin) is generally used to manage deep vein thromboembolism (DVT) and pulmonary embolism (PE). The anticoagulation mechanism of warfarin depends on its action on the vitamin $\mathrm{K}$ epoxide reductase by stopping the recycling of vitamin $\mathrm{K}$ epoxide to the reduced form of vitamin $\mathrm{K}$. The latter is an essential cofactor in the formation of active clotting factors II, VII, IX, X, regulatory factors protein C, and cofactor protein $S$ through $\gamma$-glutamyl carboxylation. In clinical practice, the different responses to warfarin due to racial differences are concerned. A normal dose for a white patient might be a dose that may cause a Chinese patient bleeding. That Chinese are more sensitive to warfarin than white Caucasians are evident by monitoring the international normalised ratio (INR) target, a laboratory test used to measure the anticoagulant effect of warfarin and serious adverse reactions. Furthermore, the duration of warfarin treatment is important to prevent recurrent venous thromboembolism (VTE) event, a challenge condition that may cause fatal outcome. The individualised strategy of warfarin treatment - the dosage and duration will be discussed through the following three cases. Case presentations Case 1: A 35-year-old White male developed a DVT without provoked causes. On admission, he presented with left calf pain, his left calf was swelling and circumference was $3.5 \mathrm{~cm}$ larger than that of the other side. The skin of his left calf was warmer and dilated superficial veins were seen. The laboratory result showed that he had high blood D-dimer level and elevated fibrinogen level. B-mode venous ultrasonography showed that he had a DVT of left popliteal vein. The patient was overweight; his lipid profile showed he had low high-density lipoprotein cholesterol level. He denied of any family history of DVT. The patient was treated by anticoagulation therapy; warfarin was initialed following 5 days of heparin. INR was monitored and aimed for the target of $2-3$. The INR target was reached when warfarin dose was $6.5 \mathrm{mg}$. After discharge the patient was followed up in the international clinic and he had no complications of bleeding. How long this patient shall take the anticoagulation medication? Case 2: A 45-year-old Chinese male developed a systemic vasculitis followed with a silent PE and then DVT. He complained with bilateral low extremities numbness and pain for 9 months on admission. Physical examination showed that he had reduced sensation of low limbs, especially the left one. Laboratory results found that his D-dimer level was elevated; high density cholesterol level was reduced. Artery ultrasonography showed he had coarse bilateral artery intima, atherosclerotic plaque in left femoral artery and artery thromboembolism in left low limb. Meanwhile venous ultrasonography showed that he had a left popliteal DVT. After diagnosis, the patient was treated by warfarin for anticoagulation, prednisolone and cyclophosphamide for vasculitis. He was quite sensitive to warfarin dose, even though the initial dose of warfarin was $2.5 \mathrm{mg}$, his INR jumped to 6.7. Warfarin was withdrawn immediately and frequent measurements of INR were done until it dropped to normal range. A smaller dose of warfarin was given cautiously. Now the patient takes $1.5 \mathrm{mg}$ warfarin every day and INR was regularly monitored. After one month treatment, repeated venous ultrasonography showed that the left popliteal thrombus was completely absorbed and the vein was recanalised thoroughly. What dose of warfarin the patient shall take? Case 3: A 45-year-old white male developed the first episode of left limb DVT due to factor V Leiden mutation in 1997. He was on medication of warfarin for years and followed up in the international clinic regularly. The dosage of warfarin was adjusted by INR monitoring, which fluctuated within 2 and 3 when his warfarin was $10-13 \mathrm{mg}$. The patient had one episode of bleeding event: a huge haematoma found by CT in posterior peritoneum after he played badminton. The emergent INR test showed that INR was 5.04, although he was on his common warfarin dose of $12 \mathrm{mg}$. How can the patient prevent the bleeding complication of warfarin? Discussion Warfarin is the most widely prescribed oral anticoagulant in the world with narrow therapeutic index and large interindividual variability. It is influenced by various clinical variables such as age, sex, genetic variations, drug interactions, food-drug interactions, and ancestral origin. Genetic variations explain the inheritance in the variation in drug efficacy and the risk of adverse drug reactions. In addition to the INR monitoring and clinical algorithms, the two major tools for physician to plan warfarin therapeutic strategy, pharmacogenomic approaches will be promising to provide alternative information to clinical utility of this drug. Despite that warfarin is tough to handle, it is still the essential drug of anticoagulation therapy for patients with DVT. The dose of warfarin should be adjusted to achieve an INR of 2 to 3 . An INR of $\geq 4$ increases the risk of bleeding complications in patients. INR varies from person to person even if the same dose is used, for some patients it can be very difficult to find a suitable dose of warfarin initially, even with frequent monitoring of the INR. In Case 2, a small induction dose of warfarin $2.5 \mathrm{mg}$ was still high for the patient; a smaller dose of $1.5 \mathrm{mg}$ was found suitable for him. On the other hand, in Case 1, INR of the patient increased slowly after heparin was given for 5 days, and an initial dose of $5 \mathrm{mg}$ warfarin did not reach the INR target. So, an extra small dose of warfarin $1.5 \mathrm{mg}$ was added and then INR target was reached. In Case 3, the dose of warfarin required for the patient was higher than the patients in Case 1 and Case 2, his dose was adjusted between $10 \mathrm{mg}$ and $13 \mathrm{mg}$. After several years of good management, this patient had an episode of bleeding complication at $12 \mathrm{mg}$, a suitable maintenance dose for him. For the three patients, racial difference is one of the underlying causes of different responses to warfarin but genetic variation might be also possible. So, it would be nice if the appropriate dose of warfarin for individual patients could be predicted before its administration. Since we had not done the pharmacogenetic tests for the three patients, we used a pharmacogenetic dosing algorithm to estimate the doses. However, even with many studies working on warfarin pharmacogenetic dosing algorithms, it is still an art to adjust the dose of warfarin by an individualised patient-oriented personalised method by experienced physicians. The calculated 
dose of warfarin $3.4 \mathrm{mg}$ in Case 2 according to the pharmacogenetic dosing algorithm was much higher than the practical dose $1.5 \mathrm{mg}$, while the calculated dose $6.39 \mathrm{mg}$ in Case 1 is close to the practical dose $6.5 \mathrm{mg}$. In clinical practice, many factors may influence the effect and the side effect of warfarin: age, height, weight, body sise, drug status that induce the liver enzyme, amiodarone status, compliance, blood pressure control, access to the hospital or clinic, economic background, previous history of various diseases, exposure to various risk factors, and some food, etc. Numerous studies verified that genetic polymorphisms of VKORC1 and cytochrome 2C9 explain the greatest variability in dose of warfarin in races such as whites, Asians and blacks. To address the concern, a combination of pharmacogenetic dosing algorism and clinical experience shall be taken into consideration when a physician treats patients of various ethnicities. Another important concern of warfarin treatment for DVT is the duration of the treatment. The goals for the initial treatment of acute DVT are control of swelling and pain, prophylaxis of recurrent VTE, and prevention of occurrence or worsening of post-thrombotic syndrome. Recurrence of VTE is a challenge risk for patients and therefore strategies of long time treatment or extended treatment were proposed. The evaluation of clinical effectiveness of initial treatment and risk factors of recurrence of VTE are important for physicians to make a right decision. Table 1 lists the risk factors of both the first episode of DVT and recurrent VTE. Some other risk factors of recurrent VTE are family history, male gender, low high-density cholesterol level, high D-dimer levels after one month anticoagulation treatment, and failure to recanalise leg vein after anticoagulation for DVT.

\section{Table 1 withdrawn}

of DVT than women. The choice of the duration of warfarin treatment is based on the consideration of the benefit-risk ratio of therapy. The benefit brought by the long-term or indefinite use of warfarin is the prevention of recurrent VTE, while the risk is the possibility of an episode of bleeding. The patient in Case 1 had an isolated calf DVT without provoked risk factors, it is recommended to assess his venous ultrasonography and D-dimer level after 3 months treatment. If his D-dimer level becomes normal and his calf vein is recanalised, he can stop warfarin as low recurrent frequency of isolated calf DVT is evident. The patient in the Case 2 had a provoked risk factor of vasculitis; however, his left calf DVT was a subsequent episode of DVT which was secondary to a silent PE, after 2 months treatment his D-dimer level was still high despite that the previous calf DVT disappeared. He should be evaluated again at the end of 3 months warfarin therapy and continue an indefinite therapy. The patient in the Case 3 had unprovoked risk of hereditary thrombophilia, his RR of recurrent VTE is 1.5 according to the guideline, so he remains on an indefinite warfarin therapy. For the two patients with extended anticoagulation therapy, adjustment of warfarin dosage should be according to the regular monitoring of INR for prevention of bleeding complications. At maintenance dose of warfarin, the incidence of major haemorrhage analysed was 22 for every 1000 patient months with the INR between 2 and 4.5. It indicates that INR monitoring should be conducted regularly but momentarily when necessary. In a word, strategy for the anticoagulation therapy for patients with DVT should be individualised by establishing the individualisation of dosage regimen of warfarin for the effectiveness and safety of antithrombosis, selecting the duration of warfarin therapy for prophylaxis of recurrence of VTE, and adjusting the dosage of warfarin for prevention of haemorrhage complications.
In a prospective cohort, Pranoni et al followed 1626 patients of proximal DVT or PE up to maximum of 10 years and found that recurrence of VTE was significantly associated with unprovoked thrombotic episodes, thrombophilia, presentation with primary DVT, short (less than 6 months) duration of anticoagulation, and aging. The total recurrent rate was $22.9 \%$, and $11.5 \%$ was fatal, either due to PE or sudden death. The cumulative recurrent incidence of DVT after discontinuation of anticoagulation increased steadily over time, approaching 7.2 (6.0 to 8.5) after 6 months, 11.0 (9.5 to 12.5) after 1 year, 19.6 (17.5 to 21.7) after 3 years, 29.1 (26.3 to 31.9) after 5 years, and 39.9 (35.4 to 44.4 ) after 10 years. The study confirmed that patients who presented with DVT of unknown origin had a more than twofold higher risk of recurrences than patients with provoked risk factors. And in the latter category of patients, those with associated medical diseases had the highest risk, while those with DVT triggered by recent trauma or surgery the lowest. Whether male gender is a risk factor of recurrence is controversy. In Prandoni's study, they didn't find that there was significant difference of recurrence in the both genders, but other studies showed that men had higher risk of recurrence 